\title{
UN PROGRAMA ON-LINE COMO RECURSO DE APOYO PARA LAS FAMILIAS RECONSTITUIDAS
}

\author{
Beatriz Triana Pérez \\ Universidad de La Laguna (Tenerife. España) \\ btriana@ull.es
}

Fecha de Recepción: 6 Febrero 2018

Fecha de Admisión: 10 Abril 2018

\section{RESUMEN}

La sociedad española está compuesta actualmente por una gran diversidad de modelos de familia. Entre ellos destacan las familias reconstituidas, cada vez más comunes, como efecto del incremento de rupturas familiares que se han producido en las últimas décadas. Una de las principales características de estas familias es la dificultad para alcanzar la cohesión entre sus miembros y poder evitar así que se produzca una nueva ruptura. Ello se debe, entre otros factores, a la complejidad de su estructura, a la idiosincrasia de las nuevas relaciones que aporta (por ejemplo, entre padrastros/madrastras y los hijastros, 0 entre hermanastros), y también al desconocimiento que tiene la sociedad sobre dichas familias y cómo desplegar dinámicas positivas en ellas. En consecuencia, se hace necesario promover acciones de intervención que ayuden a lograr su estabilidad y permanencia. La Recomendación sobre políticas de apoyo al ejercicio positivo de la parentalidad (The Council of Europe Recommendations, Rec, 2006) enfatiza la necesidad de proveer de recursos de apoyo psicoeducativo a las familias. Éstos pueden hacerse a través de cursos presenciales, 0 también mediante recursos web englobados dentro del concepto de e-parenting. El objetivo de esta comunicación es presentar los rasgos principales de un módulo de un programa basado en las nuevas tecnologías de la información para la orientación e intervención familiar, dirigido a este tipo de familias. Dicho programa se basa en la metodología del aprendizaje experiencial, donde los participantes acceden al programa de forma flexible, según su propia disponibilidad temporal. El módulo consta de dos sesiones, que aglutinan múltiples actividades, en los que se tratan contenidos relacionados con situaciones cotidianas de las familias reconstituidas que son altamente representativas.

Palabras clave: formación parental; aprendizaje on-line; familias reconstituidas 


\section{ABSTRACT}

An on-line program as support resource for stepfamilies.

Spanish society is currently made up of a large diversity of family models. Among them are stepfamilies. These are becoming more and more common, as a result of the increase in divorces in the last decades in Spain. One of the main characteristics of these families is the difficulty to achieve cohesion among their members and to avoid the occurrence of a new rupture. This is due, among other factors, to the complexity of its structure, to the idiosyncrasies of the new relationships it brings (for example, between stepparents and stepchildren, or between half-brothers), and also to the lack of knowledge that society has regarding these families and how they can develop positive dynamics. Consequently, it is necessary to promote actions that help them achieve stability and permanence. The Recommendation on policies to support positive parenting (The Council of Europe Recommendation, Rec, 2006) emphasizes the need to provide psycho-educational resources to families. This support can be applied in person, or also through web resources included within the concept of e-parenting. The objective of this communication is to present the main features of a module of a program based on new information technologies for guidance and family intervention, aimed at stepfamilies. The program is based on the methodology of experiential learning, where participants access the program in a flexible way, according to their own temporal availability. The module consists of two sessions, which include different activities, whose content is related to everyday situations of family life that are highly representative of stepfamilies.

Keywords: parental training; e-learning; stepfamilies

\section{ANTECEDENTES}

La sociedad española actual se caracteriza por estar conformada por una variedad significativa de formas familiares, además del modelo tradicional. Cada modalidad conlleva retos particulares que se añaden a los compartidos por todas ellas. Esta circunstancia hace que muchos padres y madres tengan que enfrentarse a situaciones para las que no tienen claras estrategias que guíen sus actuaciones. Este es el caso de las familias reconstituidas, modelo cada vez más común en nuestra sociedad, como efecto del incremento de rupturas familiares que se han producido en las últimas décadas. Una de las principales características de estas familias es la dificultad para alcanzar la cohesión entre sus miembros y poder evitar así que se produzca una nueva ruptura (Oliva y Arranz, 2011). Ello se debe, entre otros factores, a su complejidad estructural, a la idiosincrasia de algunas de las relaciones que engloba (por ejemplo, entre padrastros/madrastras e hijastros, o también entre hermanastros), y además, al desconocimiento que tiene la sociedad sobre dichas familias y cómo desplegar dinámicas positivas en ellas (Golombok, 2015; Triana, Plasencia y Hernández, 2009). En consecuencia, se hace necesario promover acciones de intervención que ayuden a lograr su estabilidad y permanencia.

Por otra parte, son muchos los factores que pueden llevar a algunos padres y madres a tener que enfrentar situaciones que desbordan sus capacidades y grados de seguridad ante el ejercicio parental. Tómese como ejemplos, entre otros factores, la falta de tiempo para conciliar la vida familiar y la laboral que vive la sociedad; las actuales presiones económicas; el desconocimiento sobre cómo abordar etapas algo más demandantes en el desarrollo de los hijos 0 ante transiciones particulares; la falta de conocimiento sobre el calendario evolutivo 0 acerca de estrategias parentales que permitan criar y educar con éxito a los hijos; la necesidad de atención a sus mayores o a hijos con necesidades especiales; etc. En estas ocasiones, los padres y madres requieren la obtención de múltiples apoyos que pueden ser instrumentales, emocionales o económicos, pero también de formación. Además de ello, los padres y madres son cada vez más conscientes de la importancia que tiene 
el adecuado ejercicio de sus funciones parentales, para conseguir que sus hijos alcancen niveles de desarrollo y adaptación óptimos.

Al hilo de este argumento, y en lo que se refiere concretamente a la atención de sus hijos, las políticas europeas recogen el análisis de muchas de estas realidades, y señalan la necesidad de intentar promover contextos que brinden a los hijos e hijas oportunidades adecuadas para su desarrollo. Para ello se deben movilizar todas los elementos que tengan efectos relevantes sobre la dinámica de las familias en la sociedad actual, debiendo aunar esfuerzos desde las distintas instancias sociales, políticas, culturales y económicas (Rodrigo, 2010). Con tal fin, la Recomendación sobre políticas de apoyo al ejercicio positivo de la parentalidad (The Council of Europe Recommendations, Rec, 2006) enfatiza la necesidad de proveer de recursos de apoyo psicoeducativo a las familias. Así, se pretende que los padres y madres velen por el interés superior de los menores, ejerciendo sus funciones en contextos libres de violencia, pero donde los límites y el afecto estén claramente presentes. Por otra parte, se asume un nuevo paradigma de intervención con las familias, donde se prima más la labor preventiva que la meramente asistencial, y donde se trata de enfatizar las capacidades y potencialidades parentales para ejercer como padres y madres, más que centrarse en sus déficits. En este sentido, la responsabilidad parental implica que los progenitores desarrollen relaciones positivas con sus hijos, garantizando sus derechos y tratando de optimizar su desarrollo potencial y su bienestar (Rodrigo, Máiquez, Martín y Rodríguez, 2015).

Pues bien, promover el uso de la parentalidad positiva en padres y madres se puede lograr haciéndoles participar en cursos presenciales que les aporten información, reflexión y estrategias que les sirvan de guía. No obstante, dicha meta también se alcanza mediante recursos web englobados dentro del concepto de e-parenting. El uso de las tecnologías multimedia e internet como medios de aprendizaje permiten que el usuario pueda acceder autónomamente a recursos de aprendizaje remotos, y que lo haga con plena libertad en el uso horario. Así, la participación en programas de aprendizaje online, además de permitir la posible recepción de información, también posibilita a los usuarios el poder compartir experiencias con otras personas que se encuentran en situaciones similares (Plantin y Daneback, 2009). En este sentido, a finales de la década de los noventa se empiezan a desarrollar algunas iniciativas de formación parental mediante el uso de entornos virtuales (Daneback y Plantin, 2008). Qué duda cabe que su mayor proliferación es posterior, acompañado por el mayor nivel de accesibilidad a Internet (Torres et al., 2015).

Un ejemplo de estos programas es el titulado Educar en Positivo, alojado en la página web del mismo nombre, www.educarenpositivo.es, en el que se tratan temas variados relacionados con el uso de Internet como recurso para la familia, la comprensión y guía del comportamiento infantil, la parentalidad en situaciones de tener hijos con discapacidad, la alimentación y la salud, etc. (Torres, Suárez y Rodrigo, 2014). La presente comunicación ahonda en dicho programa mediante la presentación de un nuevo módulo dirigido expresamente a las familias reconstituidas, que se titula Vivir la reconstitución familiar: retos teñidos de luces y sombras. En él se abordan múltiples contenidos que resaltan, entre otros aspectos, las especificidades y dinámicas más comunes en la reconstitución familiar, así como las estrategias que favorecen las relaciones entre sus miembros. Además, el módulo favorece que los usuarios revisen su situación familiar personal y que desarrollen compromisos de actuación que impulsen la integración y adaptación de la familia, así como la formación de una identidad como tal. Este nuevo módulo, que se describirá en el apartado siguiente, ha sido elaborado con el soporte económico concedido por el Ministerio de Economía y Competitividad al Proyecto con referencia EDU2012-38588. 


\section{OBJETIVO}

El objetivo central de esta comunicación es presentar los rasgos principales de un módulo de un programa basado en las nuevas tecnologías de la información para la orientación e intervención familiar, dirigido a miembros de las familias reconstituidas.

\section{METODOLOGÍA Y DESCRIPCIÓN DEL MÓDULO}

El módulo se basa en la metodología del aprendizaje experiencial. Ésta se soporta en el uso de situaciones educativas, extraídas de la vida cotidiana, que sirven para que los participantes analicen y reflexionen sobre dichas realidades, revisen sus propias concepciones al respecto, e impulsen cambios en sus comportamientos (Rodrigo y Palacios, 1998). Además, el módulo, al igual que el programa Educar en Positivo en su conjunto, se basa en un enfoque de los entornos personales de aprendizaje, centrado más en la persona que aprende que en la persona que instruye (Torres et al., 2015). En este sentido, se combinan actividades formativas previamente estructuradas, con otras donde los propios participantes se convierten en transmisores de información y promotores de reflexiones en el grupo. Además, el aprendizaje online ofrece la ventaja de que los participantes acceden al programa de forma flexible, eligiendo el momento de participación según su propia disponibilidad temporal.

Entrando ahora en la organización del módulo, éste consta de dos sesiones, que aglutinan múltiples actividades, en los que se tratan contenidos relacionados con situaciones cotidianas de la vida familiar, que son altamente representativas de las familias reconstituidas. La primera sesión se titula Cuando la nueva familia empieza siendo algo más que tú y yo. La sesión persigue como principal objetivo, que los participantes conozcan dinámicas familiares que ayudan a fortalecer las relaciones entre los principales protagonistas de las familias reconstituidas, y también, dinámicas que promueven el efecto contrario. La sesión se organiza en siete actividades, cuyos títulos se presentan en la Tabla 1.

\section{Tabla 1}

Título de las actividades que aglutina la Sesión 1 del programa de Reconstitución Familiar

\begin{tabular}{cl}
\hline Número de la actividad & \multicolumn{1}{c}{ Título de la actividad } \\
\hline 1 & La familia reconstituida: ¿Igual que las demás? \\
2 & Ejercer de forma exitosa como madrastra \\
3 & Ejercer de forma exitosa como padrastro \\
4 & Compartiendo experiencias con los demás \\
5 & Fortaleciendo la relación de la nueva pareja \\
6 & Yo puedo fortalecer la relación de pareja \\
7 & Recuerda \\
\hline
\end{tabular}

Se comentará, a continuación, algunos objetivos específicos perseguidos en dicha sesión. Con la Actividad 1 se pretende hacer conscientes a los participantes sobre algunos retos intrínsecos a la dinámica familiar en situaciones de reconstitución; que reflexionen sobre cuáles son sus principales preocupaciones; y que asuman la heterogeneidad y complejidad que caracteriza a dicho modelo familiar. La actividad 2 y 3 permiten, respectivamente, conocer diferentes formas de ejercer el rol de madrastra y de padrastros, y reflexionar qué aspectos de dichas actuaciones pueden contribuir a fortalecer las relaciones entre los miembros de la familia, o por el contrario, a debilitarlas. La Actividad 4 persigue el objetivo de que los participantes puedan compartir, con otras personas, las experiencias vividas como padrastros y/o madrastras, y cómo el desempeño de dicho rol ha podido afectar 
a las relaciones que sostienen con los hijos/as de su nueva pareja, destacando especialmente las estrategias que han facilitado dichas relaciones. La actividad 5 se centra principalmente en dar a conocer a los participantes el hecho de que las experiencias previas de pareja, sirven de marco de referencia para el establecimiento de nuevas relaciones, en ocasiones facilitando estas últimas, pero en otras, llegando a condicionarlas. La Actividad 6 implica el desarrollo de un compromiso por parte de cada participante para desarrollar alguna actividad que pueda favorecer la relación con su nueva pareja, atendiendo a los contenidos y reflexiones revisados en la actividad anterior. Por último, la Actividad 7 supone una breve prueba formulada con ítems de respuesta verdadero/falso que ayuda, a cada participante, a revisar el grado de aprovechamiento sobre los contenidos revisados en la Sesión 1, en relación con las orientaciones ofrecidas sobre las estrategias de actuación que se deben seguir, y las que no, para favorecer la dinámica de los integrantes de las familias reconstituidas.

La segunda sesión se titula Sentirse familia a pesar de la complejidad. En ella se amplían los temas de reflexión referidos a posibles dinámicas, tanto integradoras como disruptivas, que son habituales en muchas familias reconstituidas. En esta ocasión, los temas se centran principalmente en la relación que sostienen los progenitores con sus hijos, una vez que los primeros pasan a convivir con una nueva pareja; en la relación entre los hermanastros, esto es, entre los hijos que ha tenido cada miembro de la pareja en alguna relación anterior; y en la dificultad para generar una nueva identidad familiar. En la Tabla 2 se recogen los títulos de las actividades que aglutina esta segunda sesión.

Tabla 2

Título de las actividades que aglutina la Sesión 2 del programa de Reconstitución Familiar

\begin{tabular}{cl}
\hline Número de la actividad & \multicolumn{1}{c}{ Título de la actividad } \\
\hline 1 & La experiencia de reconstitución: ¿La viven igual todos los hijos? \\
2 & Formar una nueva familia sin resentir la relación con los hijos \\
3 & Tus hijos y mis hijos: ¿Relaciones imposibles? \\
4 & Lograr una convivencia positiva entre los hermanastros \\
5 & Desarrollar la identidad familiar: El mayor reto para los hijos \\
6 & Y nosotros, ¿qué tal lo estamos haciendo? \\
7 & Lo que es verdaderamente importante \\
\hline
\end{tabular}

Respecto a los principales objetivos específicos que se persiguen en la segunda sesión, destacan los siguientes. En la Actividad 1 se pretende que los participantes conozcan la posible heterogeneidad de percepciones y emociones con las que los hijos se enfrentan a la experiencia de la reconstitución. La Actividad 2 ayuda a reflexionar sobre qué actuaciones van a facilitar la relación parento-filial tras una reconstitución, y por el contrario, qué estrategias pueden hacer surgir e impulsar conflictos y disputas entre dichos miembros, una vez que se inicie la convivencia. Las Actividades 3 y 4 se centran en el análisis de las relaciones que se pueden dar entre los hijos que cada miembro adulto de la pareja reconstituida ha tenido en alguna relación previa. En este caso, se trata de que conozcan algunos ejemplos, pero aún más importante, que exploren y reflexionen sobre formas positivas de actuar que se relacionen con dinámicas familiares más adaptativas e integradoras. Otro tema de especial interés es el que se trata en la Actividad 5, en relación con la elaboración de la identidad familiar. Específicamente, esta actividad pretende dar a conocer a los participantes los principales factores que facilitan el desarrollo de dicha identidad. La actividad 6 intenta ayudar a los participantes a realizar una valoración sobre la dinámica que caracteriza a los distintos subsistemas de relaciones que componen su propio núcleo familiar. Por último, la Actividad 7 trata de recordar a los participantes algunos de los contenidos más relevantes tratados en la sesión, con el fin de que, a partir de dicha información, revisen las fortalezas y debilidades que caracterizan a su 
propio núcleo familiar, para ayudarles a conocer los aspectos que deben mantener y seguir impulsando, y también, aquéllos que se pueden convertir en escollos que dificulten las relaciones, para los que hay que buscar soluciones. Con tal fin, se les pide que desarrollen algún compromiso de cara al futuro para poner en práctica algunos de los aspectos que se han trabajado en la sesión.

En relación ahora con el formato que se sigue en la presentación de las actividades, todas presentan una estructura básica común. Esto es, inicialmente se presenta el título de la sesión y de la actividad, para continuar con la presentación del objetivo u objetivos que se persiguen con dicha actividad, seguido de la descripción, presentación y desarrollo de la/s estrategia/s con la/s que se va a trabajar cada objetivo. Finalmente, la actividad termina con una breve tarea de valoración personal del grado de satisfacción con la actividad realizada.

Así mismo, las distintas actividades se organizan mediante una o más tareas que ayudan al desarrollo y profundización de los objetivos y contenidos que se persiguen con cada una de ellas. Por ejemplo, entre las múltiples estrategias o tareas utilizadas, destacan especialmente el diario personal y los foros. Concretamente, el diario personal se utiliza como espacio en el que los participantes pueden reflejar pensamientos, reflexiones y experiencias personales sobre algún tema tratado en la sesión. Así mismo, la revisión del diario les permite realizar un seguimiento de su propia evolución en el transcurso de su participación en el programa. Otra tarea importante es la denominada compromiso, que supone, como la misma palara indica, el compromiso que hace el participante de desarrollar alguna actividad en su familia, con el fin de mejorar su dinámica y las relaciones entre sus miembros. Estos compromisos también se registran en el diario. Por su parte, los foros facilitan el intercambio y la expresión de opiniones y experiencias con otros participantes, y pueden llegar a convertirse en una importante fuente de apoyo para algunos de ellos, al permitirles conocer que otras personas viven situaciones parecidas a las de ellos. Los foros también les ofrecen oportunidades para conocer nuevas formas de sobrellevar algunas circunstancias. Otras estrategias o tareas pretenden aportar información relevante, en un pequeño espacio de tiempo, a modo de breves dianas informativas a considerar, o también, a modo de reflexiones finales sobre un contenido determinado, en relación con el tema trabajado. Así mismo, otras tareas se elaboran para intentar que el propio participante realice valoraciones de su propia percepción sobre la realidad que vive. Por último, la estrategia de recuerdo viene a significar una forma de auto-evaluación del manejo de los contenidos adquiridos por los participantes en el desarrollo de la sesión, a través de las distintas actividades realizadas. Dicho ejercicio se acompaña de un feed-back para que el participante, que le sirve de guía para juzgar si necesita o no realizar nuevamente alguna actividad para aprovechar mejor los objetivos que se persiguen en la sesión.

Finalmente, es importante comentar que, para poner en una determinada situación de análisis a Ios participantes, a veces se cuenta con breves vídeos que ayudan a representar experiencias variadas, algunas más positivas e integradoras y otras más de riesgo, que suelen ser comunes en las familias reconstituidas. En otras ocasiones se utilizan viñetas para ilustrar la situación concreta 0 también se usa algún breve testimonio. A partir de la información que se ofrece, se pueden presentar preguntas, o también afirmaciones que deben seleccionar sobre lo observado, para ayudarles a centrar la atención en los aspectos más sobresalientes de la situación presentada y/o sobre las posibles consecuencias de dichas actuaciones, y sus efectos sobre la relación entre los participantes. Otra estrategia consisten en pedir a los participantes que adscriban un título a cada situación que ayude a describir de forma sucinta lo observado. También se utilizada la elaboración de un puzle como medio para alcanzar información variada sobre un tema, 0 incluso, la tarea de asociar testimonios con emociones concretas que pueden derivar de las experiencias relatadas. 


\section{DISCUSIÓN Y CONCLUSIONES}

Los nuevos retos de los padres y madres para atender adecuadamente sus funciones parentales, así como su mayor conciencia de la importancia del ejercicio parental en la adaptación de sus hijos, hace que busquen nuevas alternativas de apoyo que les ayuden a desarrollar cada vez mejor sus competencias. La búsqueda de dichas mejoras debe ir dirigida hacia múltiples aspectos, tanto cognitivos, afectivos como en relación con su comportamiento (Martín et al., 2009). Sólo así se podrá conseguir que los cambios lleguen a generalizarse y a consolidarse. Además, las nuevas realidades familiares a las que se enfrentan algunos padres y madres les exigen llegar a conocer de forma más precisa los retos particulares que deben afrontar, así como las estrategias con las que pueden llegar a desplegar dinámicas más adaptativas e integradoras entre los miembros de dichas familias.

En busca de dichos objetivos se han desarrollado en España numerosos programas que exigen la presencia física de los padres y madres para su impartición. Sin embargo, ello supone un grave inconveniente teniendo en cuenta las restricciones temporales que suelen caracterizar el día a día de las familias. Por ello, las iniciativas de formación basadas en las tecnologías de la información y la comunicación (TIC) permiten superar dichas limitaciones, posibilitando al usuario acceder a la formación mediante ténicas de e-learning, en el momento que así lo deseen y tengan disponibilidad (Torres et al., 2015). En la actualidad, los padres y madres cuentan con múltiples recursos que además de proporcionar información, permiten que accedan a formatos interactivos que favorecen la comunicación y colaboración con otras personas con experiencias afines. Este intercambio contribuye a la revisión y reflexión acerca de la experiencia familiar personal, pero también, a la aplicación de los nuevos conocimientos a la vida cotidiana, fomentando así la autoconfianza en el desempeño del ejercicio parental (Castaño, Maiz y Palacio, 2008). De ahí la importancia de estos apoyos.

Por ello, el módulo de Reconstitución familiar, asociado al programa de Educar en Positivo, se convierte en una oportunidad de apoyo online para las familias reconstituidas, a través de un entorno que brinda un aprendizaje mixto que combina información más estructurada, y la aportada por el intercambio de experiencias. Esta formación es relevante en el caso de las familias reconstituidas, en las que la falta de conocimiento sobre directrices que pueden facilitar su dinámica, las hacer claramente inestables afectando a su permanencia en el tiempo. La próxima implementación del módulo que aquí se presenta, y su posterior evaluación, permitirá conocer mejor su grado de eficacia como apoyo para las familias reconstituidas. Se trata, por tanto, de resalta el papel de los programas online como una alternativa más a los programas presenciales o semi-presenciales, ya que ofrecen nuevas oportunidades de apoyo que se adaptan a las necesidades de muchas familias que conforman la sociedad española actual.

\section{REFERENCIAS}

Castaño, C., Maiz, I. \& Palacio, G. J. (2008). Prácticas educativas en entornos Web 2.0. Síntesis: Madrid.

Council of Europe (2006), Recommendation 19 of the Committee of Ministers to Member States on policy to support positive parenting. Strassbourg. Recuperado de https://wcd.coe.int/ViewDoc.jsp?id=1073507

Daneback, K. \& Platin, L. (2008). Research on Parenthood and the Internet: themes and trends. Ciberpsychology: Journal of Psychosocial Research on Cyberspace, 2, article 2. Recuperado de http://cyberpsychology.eu/view.php?cisloclanku=

2008110701 \&article $=2$

Golombok, S. (2015). Modern families: Parents and children in new family forms. Reino Unido: Cambridge University Press. 
Martín, J. C., Máiquez, M. L., Rodrigo, M. J., Byrne, S., Rodríguez, B. \& Rodríguez, G. (2009). Programas de educación parental. Intervención Psicosocial, 18(2), 121-134.

Oliva, A. \& Arranz, E. (2011) (coords.). Nuevas familias y bienestar infantil. Sevilla: Secretariado de publicaciones Universidad de Sevilla y Servicio Editorial de la Universidad del País Vasco.

Plantin, L. \& Daneback, K. (2009). Parenthood, information and support on Internet. A literature review of research on parents and professional online. BCM Family Practise, 10(1), 1-12.

Rodrigo, M. J., Máiquez, M. L., Martín, J. C. \& Rodríguez, B. (2015). La parentalidad positiva desde la prevención y la promoción. En M. J. Rodrigo (coord.), Manual práctico de parentalidad positiva (p. 25-43). Madrid: Editorial Síntesis.

Rodrigo, M.J. \& Palacios, J. (1998). Familia y desarrollo humano. Madrid: Alianza.

Torres, A., Suárez, A., Álvarez, M., Padilla, S., Rodríguez, E. \& Rodrigo, M. J. (2015). Apoyo parental online. En M.J. Rodrigo (coord.), Manual práctico de parentalidad positiva (p. 245-263). Madrid: Editorial Síntesis.

Torres, A., Suárez, A. \& Rodrigo, M. J. (2014). Educar en Positivo: Primeros resultados y retos de futuro. Revista Iberoamericana de Sistemas, Cibernética e Informática (RISCI), 11(2), 1-13. ISSN: 1690-8627 (Online).

Triana, B., Plasencia, S. \& Hernández, M. (2009). Representaciones sociales acerca de las figuras de padrastro y madrastra. Cultura y Educación, 21(4), 417-428.

Rodrigo, M. J. (2010). Promoting positive parenting in Europe: New challenges for the European Society of Developmental Psychology. European Journal of Developmental Psychology, 7(3), 281-294. 